\title{
Correction of "misunderstanding" about chorus in China: music terminology and discussion
}

\author{
1,2Hangqi Liu, ${ }^{1}$ Siyu Tian, ${ }^{3}$ Dongjun Wang, $\quad{ }^{1}$ Huibing Tan* \\ ${ }^{1}$ Jinzhou Meical University, ${ }^{2}$ Haerbin Conservatory of Music, ${ }^{3}$ Yanxin middle school, Jinan, \\ Shandong
}

In some region and community of China for culture reason, Chinese were confused for their "misunderstanding" about chorus with its diversity and performance in both of some professional experts and amateur. Chorus and related diversity are discussed among chorus, choir, signing in unison, polyphonic singing and vocal harmony as well as "grand vocal cycle" coined by Chinese etc. The term, chorus in English, is also used for singing in unison and is still dancing together in a musical. However, community group singing in unison was not considered as chorus by some Chinese conductors. By review of literatures and videos, we discussed related terms and definition. We thought that chant is useful in popular singing and community activities. Singing in unison is still a kind of normal enjoyment of music. Singing while acting and dancing is one of the ways of a chorus performance. There are also a quite few of errors among song cycle, a solo setting, and pieces of chorus, cantata and oratorio in China. We also briefly discussed that chorus is involved in types of vocal compositions and different vocal ensemble, genres, styles and nationality.

Keywords: chorus, misunderstanding, singing in unison, acting while singing, song cycle, China

* Correspondence Author, Huibing Tan, E-mai: davidtanhb@ foxmail.com No.40, Section 3, Songpo Road, Linghe District Jinzhou City, Liaoning Province, 121001, P.R.China

\section{合唱的 “误解” 中仍有误解：与中国合唱者探讨名词和术语}

Correction of "misunderstanding" about chorus in China: music terminology and discussion

\author{
1,2 刘航奇, ${ }^{1}$ 田思宇， ${ }^{3}$ 王均栋， ${ }^{1}$ 谭会兵* \\ 1 锦州医科大学, 2 哈尔滨音乐学院, ${ }^{3}$ 山东省济南市燕新中学,
}

摘要：在目前中国一些社区由于文化背景差异，对合唱存在不同的理解。有社区民众常 识的误解, 也有专业领域本身知识的欠缺, 比如: 合唱, 多声部歌唱, 齐唱, 咏唱, 怎样或 
是否需要界定, 合唱是否需用动作。本文根据文献和录像等网络资源讨论合唱的有关“误解” 问题。以说代唱的齐唱有实际应用价值, 齐唱在中国也具有普遍性, 合唱的确可以伴随动作。 鉴于有些文章把合唱体裁与独唱体裁 “声乐套曲” 混淆, 本文还讨论了 “声乐套曲”, “康 塔塔”, “清唱剧”和 “交响合唱”的关系。

关键词: 合唱; 误解, 齐唱, 动作, 声乐套曲

*通讯作者: 谭会兵: 锦州医科大学, 神经科学实验室, 音乐与脑研究课题组, 锦州医科大 学, 辽宁省锦州市凌河区松坡路三段 40 号, 邮编: 121001

本文的目的不是定义和争辩合唱, 旨在讨论有关合唱涉及的若干内容和演唱形式, 与爱 好合唱的读者进行交流。最近在网络上看到一篇文章, 《在中国, 合唱是被误解最深的高雅 艺术》, 该文章认为很多人对合唱有 3 个误解, 并对误解进行解答。

误解一: 大家合在一起唱就是合唱。

正解：合唱是集体进行多声部的演唱。

误解二：群众歌咏就是合唱。

正解：群众歌咏不同于群众合唱, 群众合唱源于群众歌咏但高于群众歌咏。

误解三: 合唱是表演艺术, 应该动起来。

正解：合唱原则上应该是一门听觉艺术，“合唱慎用动作”。

“误解” 和 “正解” 有关的观点, 有正面积极的意义, 但我们觉得有些不够全面, 需 要展开一些讨论。我们认为合唱很多是齐唱, 也可以在歌唱的时候 “动起来”, 可以带伴有 舞蹈, 而且是合唱的歌唱者歌唱的同时跳舞蹈, 甚至一定体力强度 “劲舞” 样的动作。本文 将解释合唱中带有动作既可以是一种 “高雅” 的艺术一个形式, 也有通俗的 “表演唱”。合 唱带表演有多层意思。围绕合唱还有其他的误解, 除了上述的问题, 很多人甚至把独唱的概 念和合唱混淆, 我们也提出自己的意见。

一、合唱的语言方面的解释 ( $v$. = 动词, $n .=$ 名词):

讨论这些误解需要有个参考, 探讨在中国出现的误解, 我们借助网络和国外文献开展讨 论。合唱的英文可以是 chorus 和 choir 或 choral。其中 chorus 可以翻译为 v. 合唱; 齐声 说; 异口同声地说, n. 合唱团; 齐声; 副歌; 合唱曲, 网络合唱队; 合声; 和声。chior 可 以翻译为: n. 教堂的唱诗班; 唱诗队; 公开表演的合唱团; (教堂) 唱经楼。再对” chorus “词 根词缀分析我们可以看出, 合唱起源的历史渊源或演变。

chorus 词根: chor =dance, sing, 表示"歌,舞"。

名词: 1, choir [教堂的]歌唱队, choir=chor 歌,舞 $\rightarrow$ n. [教堂的]歌唱队; 2, choreographer 舞蹈指导, choreograph[v. 设计舞蹈动作]+er 人 $\rightarrow$ n. 舞蹈指导; 3, choreography 舞蹈, 舞蹈编 排, choreograph[v. 设计舞蹈动作] $+\mathrm{y} \rightarrow \mathrm{n}$. 舞蹈,舞蹈编排; 4, chorus 合唱队; 合唱, chor 歌, 舞+us 表名词 $\rightarrow \mathrm{n}$. 合唱队; 合唱 v. 异口同声地说,随声附和。

动词: 1, choreograph 设计舞蹈动作, chor 歌,舞+eo+graph 写 [设计] $\rightarrow$ v. 设计舞蹈动作; 2, chorus 异口同声地说, 随声附和, chor 歌,舞+us 表名词 $\rightarrow \mathrm{n}$. 合唱队; 合唱 v. 异口同声地 说, 随声附和。

如果中国 “合唱是被误解” , 我们不妨再看看互联网字典（www.dictionary.com）的解 释。

Chorus, [kawr-uh s, kohr-] 
noun, plural choruses.

1. Music.

a group of persons singing in unison.

(in an opera, oratorio, etc.) such a group singing choral parts in connection with soloists or individual singers.

a piece of music for singing in unison.

a part of a song that recurs at intervals, usually following each verse; refrain.

2. simultaneous utterance in singing, speaking, shouting, etc.

3. the sounds so uttered:

a chorus of jeers.

4. a company of dancers and singers.

the singing, dancing, or songs performed by such a company.

5. a lyric poem, believed to have been in dithyrambic form, that was sung and danced to, originally as a religious rite, by a company of persons.

an ode or series of odes sung by a group of actors in ancient Greek drama.

the group of actors that performed the chorus and served as major participants in, commentators on, or as a supplement to the main action of the drama.

6. Theater.

a group of actors or a single actor having a function similar to that of the Greek chorus, as in Elizabethan drama.

the part of a play performed by such a group or individual.

verb (used with or without object), chorused, chorusing.

7. to sing or speak in chorus.

Idioms

8. in chorus, in unison; with all speaking or singing simultaneously:

They responded in chorus to the minister's questions.

总结一下 dictionary.com 定义, 解释第一条, 一群人在一起齐唱, 第二条, 合唱是分声 部。其他有合唱伴有舞蹈和演员。由此, 我们理解合唱首先是齐唱, 合唱可以伴有动作。

百度百科的释义指出（http://baike.baidu.com/item/\%E5\%90\%88\%E5\%94\%B1/3002):

(1).音乐用语。两组以上的歌唱者，各按本组所担任的声部演唱同一乐曲。分同声与混 声两种: 同声的由男声或女声单独组成; 混声的由男声和女声混合组成。按声部的多少, 可 分二部合唱、三部合唱、四部合唱等。王西彦《古屋》第三部七: “在继续而来的歌咏上, 他们合唱的声音是那样雄壮而宏大。”

(2).音乐用语。指单声部的齐唱。朱自清《中国歌谣・歌谣的起源与发展》: “只在唱和 曲时，才大家合唱。”

从这些文字的解释理解，合唱是可以齐唱。国内和国外的实际情况，我们再具体讨论合 唱的有关实际演唱形式。

\section{二、有关合唱的形式: 术语和解释}

1, 合唱是不是可以齐唱

音乐可以是单旋律乐曲或单音音乐 (monophony), 合唱可以是齐唱（unison）。一位歌手 演唱或一件乐器演奏, 而没有伴奏和声或和弦, 当然一个单一旋律的织体音乐也可以有一 
个诗班或合唱团演唱以同一音高齐唱, 也可以相差一个八度演唱。另外, 如果一个旋律由 2 或更多的乐器一起以固定的音高, 比如纯五度演奏, 仍然可称为单旋律乐曲, 我们不妨给这 种齐唱叫双声部齐唱 (two-part unision)。素歌 (Plainsong), 来自中世纪早期教堂音乐, 或 圣歌; 单声圣歌; 单声颂歌, 也称为 plainchant (拉丁语: cantus planus)。素歌是单声部组成 的单一的旋律乐谱, 通常它的节奏比较自由, 不像现在有节拍西方音乐的节奏。著名的咏唱 有以教皇格利高里命名的无伴奏齐唱圣歌 (格列高利圣咏, 圣歌, Gregorian chant)。之后到 了中世纪后期, 写于 1250 年左右作品” Sumer Is Icumen In"(夏日来临)是一部多声部 (polyphony) 轮唱作品, 有 6 个声部。多声部就是有独立的 2 个线条以上音乐组成的音乐。 由此演唱的作品就是和声合唱。

上述谈到咏唱(Chant)的歌唱, 可派生出至少 15 种歌唱种类或功能类别形式, 比如祷告

(Prayer)，诗篇 (Psalm)，赞美诗 (Hymn)，唱 (Antiphon)，唱和 (Responsory)，引唱 (Introit)， 哈来路亚 (Alleluia)，供奉 (Offertory) 和交流 (Communion) 等。这些 Chant 可以齐唱, 也 可以多声部合唱。我们非常熟悉的奥尔夫的《布兰诗歌》 (Carmina Burana) 就与 Chant 有渊 源, 其中 veris leta facies（春意盎然）长达 3 分半以上的歌唱, 以八度的 Chant 样的齐唱。 所以说齐唱可以是合唱。有些教堂的赞美诗很多没有编印成多声部, 教友也就是自然地齐唱。 这种谱子也会给没有条件分声部的教堂齐唱用, 可以为 Unison Choir (齐唱诗班, 或齐声合 唱)。还有给儿童的合唱乐谱也可以是齐声合唱。

\section{2, 齐唱应用的普遍性和社会功能}

生活中, 劳动号子有齐唱, 也有多声部。在西方, 齐唱的生活中用的比较多的场合是教 堂, 因为每星期天礼拜都需要唱, 合唱是必须的内容。礼拜中歌唱是宗教活动的一部分, 其 中的合唱是仪式性, 程式化东西, 不是以娱乐为出发点, 合唱的作用和目的是表达崇敬和爱, 已及表述内心的想法, 信念和彼此共同感受、感情或心情。除了诗班唱的赞美诗 (anthem), 一般需要全体起立唱赞美诗(hymn)或 chant 就有 3-6 次。一本常用的赞美诗集可以多达 800 多首, 就算一年有 60 次教堂聚会和礼拜, 5 乘 60, 不重样的唱, 一年只能唱 300 首; 所以 除了诗班的用和声合唱, 其他多数人还是齐唱。除了每周固定礼拜, 遇到圣诞节教堂常规还 要有几种多次活动, 教友没有时间排练, 而且很多教堂需要礼拜的内容是唱出来的。去教堂 参加礼拜和聚会, 没有一个礼拜的内容是相同的, 需要合唱的内容也不用有排练的时间, 多 是随手拿出座位边放置好的赞美诗集, 按当天的礼拜册规定的唱。除了规定赞美诗, 教堂还 会有临时加入的需要以唱代说的内容, 这就是 chant 的作用。另外很多宗教以外场合没有歌 谱, 也是只能齐唱。

3, 合唱是群众性的歌唱活动。

合唱乐团或组合（Vocal ensembles）的英文来自于拉丁 "vocalis" - 'loud（大声）', 'singing (歌唱)', 然而演唱的艺术在于控制。向延生在《中国合唱百年事略》提到齐唱在中国合唱 发展其到重要作用。“群众” 提法也可能仅限于中国。计划体制下 “群众” 合唱团等于非专 业组织。界定 “群众” 合唱和专业合唱, 关键是演唱曲目和水平, 中国一些专业的合唱团比 不上国外的民间合唱团。

在西方合唱基本上群众性的, 很多合唱团的成员是以业余人员为主。童声合唱是《战争 安魂曲》这样作品的必要声部, 甚至一个普通小镇的群众 “咏唱” 或合唱, 水平也不差。我 们用足球做间接的解释, 2016 欧洲杯就像只有 30 多万人口的冰岛足球队可以踢败英国足球 队; 2016 年 01 月 15 日足球 U23 亚洲杯小组赛, 临时组件的国破家亡颠沛流离的叙利亚足 球队可以打败中国队。群众合唱, “低级” 的合唱, 比如 chant; Gregorian chant 有艺术价值, 
可以为最好的唱片公司灌制录音出版。

另外, 国外识谱是一种文化, 识谱比例大于中国, 其多声部合唱音乐或混声合唱容易组 织, 但是他们也仍有齐唱。国外中小学有合唱科, 中学生参加音乐剧, 演出整场一部音乐剧 也是正常教学或学校俱乐部活动, 中学生报名参加歌剧艺术节或学习班也很正常。音乐学院 声乐专业选修舞蹈可非常自然。在美国和加拿大, 如果声乐歌手不能经常签约独唱角色, 自 然要参加或争取参加合唱, 如果在包括合唱表演中不能跳舞, 演员的职业发展就受到限制。 在中国, 专业团体的合唱团体, 很多队员不识五线谱, 甚至某些团体的领导也没有必要音乐 基础知识。所以关于合唱和有关声乐知识的也有会误解。

\section{二、合唱可以带表演, 带动作}

民间劳动号子和各种节日歌咏, 很多是伴有动作，和舞蹈。

有一种合唱带表演叫理发店合唱 (barbershop chorus), 为无伴奏和声法演唱形态, 可以 四重唱, 小型团队演唱 12 人, 也可以是大型团队合唱 150 人, 声音不同于声乐, 比较通俗。 美国加拿大有相应的协会 Barbershop Harmony Society, 英国也有相应的组织 British Association of Barbershop Singers, 也有国际比赛 International Chorus Champions 。他们可以 是男声 barbershop, 男士西装革履, 但是不妨碍他们一边合唱一边运用肢体语言进行合唱表 演, barbershop music 比赛很多是带动作的, 甚至指挥也可以动, 可以舞, 在舞台上满场舞 动也不奇怪。还有很多动作需要体力和强度很到技术, 如打旋, 翻跟头, 叠罗汉。另外, 合 唱队员可以计入道具, 比如挥动旗帜, 戴装饰项链或手舞彩巾等。歌声讲究动态伸展, 声音 夸张, 有很多甩音, 制造自己独特音色, 比如铃响和声 (ringing chord)。除了传统曲目, 他 们很多是原创, 自创或改编的曲目, 比如百老汇, 声乐爵士 (Vocal Jazz), 欧美流行组合的 先声"嘟 - 喔普"之声(Doo-Wop), 或格什温（Gershwin）到披头士（the Beatles）。也有女声 barbershop。这是通俗的带有表演的合唱。当然还有其他的带表演合唱。比如福音诗班 (Gospel choirs），表演合唱（show choir），声乐爵士合唱（vocal jazz choir）。教堂里的福音诗班有 一定表演, 比如走动, 还有肢体打手语边唱边打手语的肢体敬拜。中小学生的某些表演, 尤 其是幼儿园和小学, 肢体语言 (gesture)学习节奏和拍节。当代 choir, 有摇滚 choir。

还有一种音乐剧 (musicals, musical theatre), 合唱带有表演, musicals 可以是百老汇, 好莱坞和其他的电影, 这里的合唱就是表演的一部分重要的是。相对于歌剧, 音乐剧首先是 演员, 然后才是歌手和舞蹈演员, 可以说其中的合唱是表演唱, 与表演融为一体, 所以音乐 剧在中国又叫歌舞剧。由于翻译和理解的差异, 中文有时也可以反过来叫歌舞剧为 opera, 但是即使这样谈论的内容还是音乐剧。还有芭蕾舞中的合唱是为表演服务, 在另一种形式上 的融合。百老汇和好莱坞 (Hollywood) 或电影音乐剧 (musical films), 有些曲目或段落可以 在北美的歌剧大师班上作为教学内容, 所以合唱完全也可以是高雅的表演合唱。歌剧中的合 唱当然是有动作, 是不是高雅艺术自然就无需解释了。虽然音乐剧就可以是百老汇或电影的 节目, 然而音乐剧最重要的特点在由业余爱好者或中学或大学生们在教堂, 学校, 社区剧院 演出。绝大多数中学有相应俱乐部, 爱好表演又能唱的学生一般有排演一部音乐剧非常正常。 中学, 大学毕业还想演出, 就进入教堂或社区相应组织协会或社团。简单用钢琴伴奏, 有讲 究的找个小型交响乐队也不是很难。由此演出场地, 演员, 歌手, 剧务人员, 服装, 道具, 布景不需要政府资助, 国外藏富于民, 老百姓自己可以解决。为保证演出水平, 这些演出都 会专业人士指导。

歌剧不像音乐剧, 里的合唱可以有表演动作。合唱是听觉艺术, 合唱队员演唱时可不可 以加动作, 可不可以有表演动作的确要看场合。歌唱本身是听觉艺术, 但是放在现场的舞台 上, 银幕中就需要歌唱者需不需要表演。 
另外有些电影就是用 chorus（合唱）或借用 “合唱 “一词来命名，其内容就有合唱加 舞蹈场面。比如电影《歌舞线上 A Chorus Line (1985)》。我们也必须承认虽然, 有些合唱的 原唱或作为戏剧, 电影, 音乐剧或歌剧演出带有表演或动作, 把这些合唱单独拿出来, 在古 典音乐会中, 还是不需要动作的进行演出。

自然和很多合唱也的确是没有动作的, 比如（声乐）赞美诗（anthem）（中文翻译是作 者为便于区分 hymn 和实际演唱体会临时启用), 赞美诗 (hymn), 康塔塔 (cantata), 清唱 剧 (oratorio), 弥撒 (Mass), 安魂曲 (Requiem)。还有合唱交响乐 (choral Symphony)。

\section{四、合唱体裁与独唱体裁 “声乐套曲”混淆}

合唱在歌剧和音乐剧里是一个该体裁音乐作品的组成部分。我们下面要讨论以合唱为主 题或合唱是主要结构的音乐。

1, 声乐套曲(song cycle)的误解和误用

我们首先确定 “声乐套曲” 是诗歌与钢琴构成的独唱音乐作品, 下面讨论, 还会提到。 有些文章, 在表述合唱作品时, 对合唱体裁概念有些混淆的表述。比如有一篇关于《神 话中国》的研究论文, 有这样的文字表述: “而在中国大型交响合唱声诗《神话中国》诞生 之前, 中国没有关于神话故事和传奇为题材的大型声乐套曲, 所以《神话中国》的诞生, 对 于中国音乐的发展”, “中国最早的大型声乐套曲, 是 1932 年黄自作曲清唱剧的《长恨歌》

“, “音乐节专家权威欣喜地看到了 cantata（康塔塔）中国式、民族化以后应该呈现的形态 和面貌, 中国各个普通合唱爱好者就需要慢慢的认识《神话中国》的艺术价值”。“大型声 乐套曲”, “清唱剧”, “康塔塔” 是不同体裁的音乐体裁。我们觉得《神话中国》不是声乐套 曲, 也不是康塔塔样的作品, 应该对应的是清唱剧。另外, 上面提到的 “交响合唱音诗” 我 们一时找不到对应的术语。有一个接近的名词“音乐交响诗”, 一种单乐章的标题交响音乐, 脱胎于 19 世纪的音乐会序曲, 强调诗意和哲理的表现。然而 “交响合唱音诗” 显然不是指 单乐章的合唱了，所以这个提法仍然有使读者误解的地方。

\section{2 , 百度百科的解释}

查看网络, 百度百科对这些概念存在自相矛盾的地方。

百度百科关于 “声乐套曲” 的注解：

“声乐套曲一般的特点是: 在统一的标题之下, 用若干首歌曲来表达同一主题。套曲中 的各首歌曲可独立成曲, 彼此的表演形式也往往不同 (如分独唱、重唱、齐唱、合唱等)。...”

(本文作者认为百度百科解释是错误的)

“中国 …..著名的《黄河大合唱》、《长征组歌》等, 其音乐结构形式都属于声乐套 曲。”(声乐套曲是独唱音乐)

“西方声乐套曲, 它的原意为 “环圈舞”, 即: Song-cycle。声乐套曲是利德盛期, 确定下来的一种固定的歌曲套曲形式。声乐套曲中的各首歌曲通过其内容和音乐风格, 使它 们之间相互联系在一起。声乐套曲这种形式不仅是贝多芬的首创, 而且他 1816 年根据诗人 阿洛伊斯、耶特莱斯的 6 首诗创作的《致远方的爱人》被公认为是德国第一部声乐套曲。”

（本文作者确定百度百科这个解释是正确的, Song-cycle 其实英文的术语, 因为对应的德语 是 Liederkreis or Liederzyklus, 因为声乐套曲首先是贝多芬创作 Reihe (series 系列), Kranz (ring 环), Zyklus (cycle 循环, 套曲) or Kreis(circle 圆圈), 所以我们没有看到 Song-cycle 词组内有“舞” 的意思)。

“康塔塔（意译为清唱套曲）（Cantata）是一种包括独唱、重唱、合唱的声乐套曲, 一 般包含一个以上的乐章, 大都有管弦乐伴奏, 与中国的大合唱体裁特点十分相近, 因而一度 被误译为大合唱。”(本文作者认为康塔塔不是声乐套曲) 
“清唱剧是一种大型套曲结构, 有一定的戏剧情节, 由多种声乐曲以及管弦乐队组成, 其中包括咏叹调、宣叙调、重唱、以及合唱, 是介于歌剧和康塔塔之间的多乐章大型声乐套 曲, 由管弦乐队伴奏。”（本文作者认为清唱剧不是声乐套曲）

以看到百度百科对 “康塔塔” “清唱剧”与上述的西方 “声乐套曲” 自相矛盾。

我们注意到互联网对声乐套曲还有其他并列的名称联篇歌曲或组歌。

3, 专业领域研究论文中概念误用

声乐套曲作为音乐体裁, 专业领域有的人清楚有的混淆。我国作曲家郑秋枫创作声乐套 曲《祖国四季》是独唱作品, 绝大多数人认可这部独唱作品体裁是声乐套曲, 和认为舒伯特 《冬之旅》(Die Winterreise) 是声乐套曲相一致。可是, 在实际谈及合唱作品时, 声乐套曲 就会误用。彭灵的硕士论文, 在分析郑秋枫的声乐套曲《祖国四季》时, 却在比较中西方声 乐套曲差异时, 错误地将《长征组歌》归类为声乐套曲。杨捷在其论文 “《黄河大合唱》与 清唱剧《长恨歌》之比较研究”, 错误的将清唱剧（oratorio）翻译成 cantata。陈巧巧在 “陈 田鹤清唱剧《河梁话别》和艺术歌曲研究”, 错误认为 “清唱剧是起源于西方的多乐章大型 声乐套曲” (实际上声乐套曲创作晚于清唱剧)。刘子锐也错误地将康塔塔认为是声乐套曲, “原来也是单声部风格的产物”, 不过在 “民族化的康塔塔” zhong 中有正确地指出 “洗星海 的《黄河大合唱》, ....属于康塔塔的一种一一大型康塔塔”。除了合唱中出现误解和概念错误, 关于艺术歌曲也有类似的情况出现。把声乐套曲和场合体裁的混淆是不严谨的。犹如陆在易 认为“艺术创作的规律之一就是限制。如果没有限制也就没有体裁之分了。把声乐套曲作为 形容词解释合唱作品, 还有诸如 “康塔塔 (意译为清唱套曲) (Cantata) 是一种包括独唱、 重唱、合唱的声乐套曲” 是一种很不严谨的 “张冠李戴”误用。邹仲之翻译出版《欧洲声乐 作品译文集》 (一、二集) 收录 32 为作曲家 38 部声乐套曲和 36 部形式多样的大型声乐作 品, 对声乐套曲以外的合唱作品体裁有清楚的示范性归类。

4，中国人杜撰的名词 “大合唱” (grand vocal cycle)

我们注意到一些中国人对合唱还有其他有别于西方的自己理解, 有人称为 “中国人对声 乐套曲的理解”。比如 “大合唱”, 还有随之命名的英文术语 grand vocal cycle。这是词源学问 题, 我们暂时不在这里赘述。我在理解“grand=大”, “vocal=声乐”, “cycle=套曲=合唱”。其百 度百科的解释是: “大合唱指集体演唱多声部声乐作品的艺术门类”。“包括独唱、重唱、对 唱、齐唱与合唱（有时穿插朗诵）的, 通常由管弦乐队伴奏的多乐章的大型声乐套曲” (百 度百科又一次混淆概念, 我们再次指出声乐套曲是独唱音乐作品)。但是其中的解释有不严 谨的地方, 因为 “声乐套曲” 对应的 song cycl。声乐套曲是独唱作品。声乐套曲和艺术歌曲 有关联。艺术歌曲（art song，德语 Leid）是诗歌与钢琴的相结合的音乐作品体裁。多首诗歌 组成, 具有关联的情景或情节或故事, 配合钢琴写成的音乐作品。最初是贝多芬首先创作《致 远方的爱人》。中文的很多学术文章和网络资料也明确指出伯特声乐套曲《冬之旅》和《美 丽的磨房姑娘》是声乐套曲。

在时间规模上看, 是不是加了 “大型” 两字, “大型声乐套曲” 针对作品演出时间很长 呢? 伯特声乐套曲《冬之旅》和《美丽的磨坊女》这样独唱作品, 演唱需要 1 小时 10-20 分 钟左右。《黄河大合唱》也不超过 40 分钟, 而我们在有些地方可以看到对应翻译是 cantata(康 塔塔)。布里顿《战争安魂曲》演出也超过 1 小时 20 分钟, 威尔第《安魂曲》 1 小时 20-30 分钟。亨德尔的《弥撒亚》如果不裁剪演唱时间可能长达近 3 小时。我们不是单指《黄河大 合唱》名字中的 “大合唱” 有什么不妥, 我们认为很多中国的声乐合唱称为 “大合唱” (grand vocal cycle）不过贴切。康塔塔和清唱剧的区别之一就是, 后者比前者演唱时间长, 合唱人 数和乐队规模大, 相对康塔塔, 清唱剧本身有 “大的康塔塔” 意思。其实《黄河大合唱》 对应称为 oratorio(清唱剧), 完全可以对号入座地为这部作品找到对应体裁位置, 现在又杜 撰出来一个 “大合唱” 的概念或称呼, 相比很多西方的经典合唱作品, 被称作 “大合唱” 的 
作品, 实际上还只能算 “中等合唱”。因为论合唱的技术规模大小，中国的清唱剧都不算大。 西方还有一种音乐需要双或多合唱团 (double (or multiple) choirs), 他们每一个合唱在一起, 但又是在意义明显彼此分开。这种合唱是 16 世纪的一种演唱, 特定的名称叫威尼斯多重合 唱 (Venetian polychoral style), 比如布里顿《战争安魂曲》, 才算大一些的合唱。由于语言 学的问题, “大合唱” 其实是清唱剧, 时间短小的合唱为康塔塔。

应贴切地使用 “大合唱”，否则只是文字符号：“大+合唱”。马勒的第八交响曲（千人交 响曲), 由于交响乐队和合唱团的庞大, 有人提出一个新概念称为 “新交响宇宙 (new symphonic universe)” (马勒本人不以为然), 形容这部作品为康塔塔清唱剧赞美诗艺术歌曲的 合成交响乐 (a synthesis of symphony, cantata, oratorio, motet, and lied in a combination of styles), 声乐部分有 2 个四声部合唱团(SATB), 一个童声合唱团, 8 个独唱, 171 人组成的交响乐团 以及 850 人的合唱队, 总共需 1029 人参加演出。对比中国 “大合唱” 的冠名, 失之偏颇。

\section{五、结语}

对合唱的误解，我们谈及了齐唱（unison），咏唱（chant），合唱（chorus，choir, choral， polyphonic singing or vocal harmony），康塔塔（cantata）,清唱剧（oratoria）,声乐套曲（song cycle），艺术歌曲（art song）, 还有中国人自己的 “大合唱”（grand vocal cycle）。造成这样的 困惑可能是因为词源学 (etymology) 的原因 (我们将另写文章论述)。

普及对多声部合唱和混声合唱知识利于提高社区合唱水平。对于合唱的误解, 有体制和 音乐教育方面的问题。然而象 “声乐套曲” 是独唱作品与合唱作品混淆是专业人士责任问题。 音乐是发展的, 各种形式和体裁也不是在创建初期就定义的准确。就像合唱交响乐（choral Symphony), 是柏辽兹为他的首次定义, 然而这种音乐题材早在贝多芬的第九交响曲就确立 了。尽管对合唱存在不同观点, 希望通过 “误解” 和 “正解” 讨论, 起到普及多声部合唱的 知识, 对促进合唱有积极作用。相信很多合唱组织能够自如地驾驭各种形式演唱, 并在创作 中灵活地应用。

\section{参考文献:}

1. 陈馥珊，音符的简史，音乐爱好者 2017-01-10, p42-45

2. 张又尹，大型交响合唱音诗《神话中国》合唱的表现特点论文，硕士学位论文， 2014

3. 王真, 浪漫主义时期声乐套曲的文化阐释, 中国音乐学[J], 2007-10-15,p124-126

4. 板俊荣, 浅谈舒伯特三部声乐套曲中的调性布局与局部的调性变化 南京艺术学院学 报(音乐及表演版) [J], 2002-08-25

5. 彭灵, 郑秋枫的声乐套曲《祖国四季》的演唱探析四川师范大学, 硕士学位论文[D], 2010-04-01,

6. 郑欢, 声乐套曲《祖国四季》的创作特征分析, 黄河之声[J] 2011-03-23

7. 杨捷, 《黄河大合唱》与清唱剧《长恨歌》之比较研究, 河北大学[D] 2002-10-01

8. 陈巧巧, 陈田鹤清唱剧《河梁话别》和艺术歌曲研究, 温州大学, 硕士学位论文[D], 2013-03-01, p4

9. 刘子锐; 杨杰, 康塔塔及其流变初探, 中国石油大学胜利学院学报 [J], 2015-03-15, 29 (1) :53-55

10. 梁茂春, 论 “文革” 时期的艺术歌曲, 中央音乐学院学报 [J], 2008-02-15

11. 徐兆仁, 浅议艺术歌曲的属性与德奥艺术歌曲的特点乐府新声, 沈阳音乐学院学报 [J] 
2005-06-30 p43-47

12. 邹仲之，欧洲声乐作品译文集（上下)，上海音乐学院出版社[M]，2011

13. 王大燕，艺术歌曲概论，上海音乐出版社 [M]，2009,2

14. 苏玲芬, 黄进, 中外艺术歌曲理论研究与演绎探讨, 中国书籍出版社 [M], 2016, p16

15. 王晶, 王晓北, 浅析我国多声部民歌中的支声复调因素, 艺术教育, 2006-08-01, p8283

16. 樊祖荫, 中国多声部民歌的织体形式研究, 艺术探索, 1991-07-02, p1-10

17. 樊祖荫,论支声音乐,中国音乐,1983-04-02，p22-25

18. 樊祖荫,论汉族的多声部民歌,音乐艺术，1988-04-01 p26-31，p63

19. Averill, Gage (2003). Four Parts, No Waiting: A Social History of American Barbershop Harmony. Oxford University Press. p. 164 and 166. ISBN 0-19-511672-0.

20. Roger Kamien. An appreciation Music, Fourth brief edition. McGraw-Hill Higher Education. p65,86,p130-143 p215-221, p401 [M]

21. Roger Kamien. Music in the middle ages, An appreciation Music, Fourth brief edition. McGraw-Hill Higher Education. p65-221,p401 , [M]

22. Frank Granville Barker and Riccardo Muti.Recital and chamber music, song, Music., Winward. 1981. p264-270 [M]

23. Willian Mann, James Galway's Music in time, The Lied. Harry N. Abrams, Inc., Publishers.1983.p116-119[M]

24. Robert Ainsley, The ultimate encyclopedia of classical music. Carlron Books Limited. 1995, p206-247[M]

25. Joseph Machilis and Kristine Forney, The Enjoyment of Music, Seventh edition, Norton, p73,p374-391, p410-412

26. Alan Kendall, 5. Romanticism, Lieder and religious music. Music, Its story the west[M].p167-173 